\title{
Safety and efficacy assessment of allogeneic human dental pulp stem cells to treat patients with severe COVID-19: structured summary of a study protocol for a randomized controlled trial (Phase I / II)
}

Qingsong Ye ${ }^{1 \dagger}$, Hua Wang ${ }^{2+}, \mathrm{Xia}_{\mathrm{Xia}}{ }^{3+}$, Chenliang Zhou' ${ }^{1}$ Zhiming Liu', Zun-en Xia', Zhan Zhang ${ }^{1}$, Yang Zhao', Jun Yehenala', Si Wang ${ }^{1}$, Gangqiao Zhou ${ }^{2}$, Ke Hu${ }^{1}$, Bin Wu ${ }^{4}$, Chu-Tse Wu ${ }^{2^{*}}$, Songling Wang ${ }^{5^{*}}$ and Yan He ${ }^{6,7^{*}}$ (D)

\footnotetext{
Abstract

Objectives: To assess the safety and therapeutic effects of allogeneic human dental pulp stem cells (DPSCs) in treating severe pneumonia caused by COVID-19.

Trial design: This is a single centre, two arm ratio 1:1, triple blinded, randomized, placebo-controlled, parallel group, clinical trial.

Participants: Twenty serious COVID-19 cases will be enrolled in the trial from April 6th to December 31st 2020. Inclusion Criteria: hospitalised patients at Renmin Hospital of Wuhan University satisfy all criteria as below:

1) Adults aged 18-65 years;

2) Voluntarily participate in this clinical trial and sign the "informed consent form" or have consent from a legal representative.

3) Diagnosed with severe pneumonia of COVID-19: nucleic acid test SARS-CoV-2 positive; respiratory distress (respiratory rate $>30$ times / min); hypoxia (resting oxygen saturation $<93 \%$ or arterial partial pressure of oxygen / oxygen concentration $<300 \mathrm{mmHg}$ ).

4) COVID-19 featured lung lesions in chest $X$-ray image.
}

Exclusion Criteria: Patients will be excluded from the study if they meet any of the following criteria.

(Continued on next page)

\footnotetext{
*Correspondence: 139100026365@163.com; slwang@ccmu.edu.cn; helen-

1101@hotmail.com

${ }^{\dagger}$ Qingsong Ye, Hua Wang and Xia Xia contributed equally to this work.

${ }^{2}$ Beijing Institute of Radiation Medicine, Beijing 100850, China

${ }^{5}$ School of Stomatology, Capital Medical University, Beijing 100006, China

${ }^{6}$ Tianyou Hospital, Wuhan University of Science and Technology, Wuhan 430064, Hubei, China

Full list of author information is available at the end of the article
}

(c) The Author(s). 2020 Open Access This article is licensed under a Creative Commons Attribution 4.0 International License, which permits use, sharing, adaptation, distribution and reproduction in any medium or format, as long as you give appropriate credit to the original author(s) and the source, provide a link to the Creative Commons licence, and indicate if changes were made. The images or other third party material in this article are included in the article's Creative Commons licence, unless indicated otherwise in a credit line to the material. If material is not included in the article's Creative Commons licence and your intended use is not permitted by statutory regulation or exceeds the permitted use, you will need to obtain permission directly from the copyright holder. To view a copy of this licence, visit http://creativecommons.org/licenses/by/4.0/. The Creative Commons Public Domain Dedication waiver (http://creativecommons.org/publicdomain/zero/1.0/) applies to the data made available in this article, unless otherwise stated in a credit line to the data. 
(Continued from previous page)

1. Patients have received other experimental treatment for COVID-19 within the last 30 days;

2. Patients have severe liver condition (e.g., Child Pugh score $>=C$ or AST $>5$ times of the upper limit);

3. Patients with severe renal insufficiency (estimated glomerular filtration rate $<=30 \mathrm{~mL} / \mathrm{min} / 1.73 \mathrm{~m}^{2}$ ) or patients receiving continuous renal replacement therapy, hemodialysis, peritoneal dialysis;

4. Patients who are co-infected with HIV, hepatitis B, tuberculosis, influenza virus, adenovirus or other respiratory infection viruses;

5. Female patients who have no sexual protection in the last 30 days prior to the screening assessment;

6. Pregnant or lactating women or women using estrogen contraception;

7. Patients who are planning to become pregnant during the study period or within 6 months after the end of the study period;

8. Other conditions that the researchers consider not suitable for participating in this clinical trial.

Intervention and comparator: There will be two study groups: experimental and control. Both will receive all necessary routine treatment for COVID-19.

The experimental group will receive an intravenous injection of dental pulp stem cells suspension $\left(3.0 \times 10^{7}\right.$ human DPSCs in $30 \mathrm{ml}$ saline solution) on day 1,4 and 7;

The control group will receive an equal amount of saline (placebo) on the same days.

Clinical and laboratory observations will be performed for analysis during a period of 28 days for each case since the commencement of the study.

Main outcomes: 1. Primary outcome

The primary outcome is Time To Clinical Improvement (TTCl). By definition, $\Pi \mathrm{TCl}$ is the time (days) it takes to downgrade two levels from the following six ordered grades [(grade 1) discharge to (grade 6) death] in the clinical state of admission to the start of study treatments (hDPSCs or placebo).

Six grades of ordered variables:

\begin{tabular}{ll}
\hline Grade & Description \\
\hline Grade 1: & Discharged of patient; \\
Grade 2: & Hospitalized without oxygen supplement; \\
Grade 3: & Hospitalized, oxygen supplement is required, but NIV / HFNC is not required; \\
Grade 4: & Hospitalized in intensive care unit, and NIV / HFNC treatment is required; \\
Grade 5: & Hospitalized in intensive care unit, requiring ECMO and/or IMV; \\
Grade 6: & Death. \\
\hline
\end{tabular}

Abbreviations: NIV, non-invasive mechanical ventilation; HFNC, high-flow nasal catheter; IMV, invasive mechanical ventilation.

\section{Secondary outcomes}

2.1 vital signs: heart rate, blood pressure (systolic blood pressure, diastolic blood pressure). During the screening period, hospitalization every day (additional time points of D1, D4, D7 30min before injection, 2h \pm 30min, $24 \mathrm{~h} \pm 30 \mathrm{~min}$ after the injection) and follow-up period D90 \pm 3 days.

2.2 Laboratory examinations: during the screening period, 30 minutes before D1, D4, D7 infusion, 2h $\pm 30 \mathrm{~min}$, $24 \mathrm{~h} \pm 30 \mathrm{~min}$ after the end of infusion, D10, D14, D28 during hospitalization or discharge day and follow-up period D90 \pm 3 days.

2.3 Blood routine: white blood cells, neutrophils, lymphocytes, monocytes, eosinophils, basophils, neutrophils, lymphocytes, monocytes, eosinophils Acidic granulocyte count, basophil count, red blood cell, hemoglobin, hematocrit, average volume of red blood cells, average red blood cell Hb content, average red blood cell $\mathrm{Hb}$ concentration, RDW standard deviation, RDW coefficient of variation, platelet count, platelet specific platelet average Volume, platelet distribution width,\% of large platelets;

2.4 Liver and kidney function tests: alanine aminotransferase, aspartate aminotransferase, alkaline phosphatase, y-glutamyl transferase, prealbumin, total protein, albumin, globulin, white / globule ratio, Total bilirubin, direct bilirubin, cholinesterase, urea, creatinine, total carbon dioxide, uric acid glucose, potassium, sodium, chlorine, 
(Continued from previous page)

calcium, corrected calcium, magnesium, phosphorus, calcium and phosphorus product, anion gap, penetration Pressure, total cholesterol, triacylglycerol, high density lipoprotein cholesterol, Low density lipoprotein cholesterol, lipoprotein a, creatine kinase, lactate dehydrogenase, estimated glomerular filtration rate.

2.5 Inflammation indicators: hypersensitive C-reactive protein, serum amyloid (SAA);

2.6 Infectious disease testing: Hepatitis B (HBsAg, HBsAb, HBeAg, HBeAb, HBcAb), Hepatitis C (Anti-HCV), AIDS

(HIVcombin), syphilis (Anti-TP), cytomegalovirus CMV-IgM, cytomegalovirus CMV-lgG; only during the screening period and follow-up period D90 \pm 3.

2.7 Immunological testing:

Collect peripheral blood to detect the phenotype of T lymphocyte, B lymphocyte, natural killer cell, Macrophage and neutrophil by using flow cytometry.

Collect peripheral blood to detect the gene profile of mononuclear cells by using single-cell analyses.

Collect peripheral blood serum to detect various immunoglobulin changes: IgA, lgG, IgM, total lgE;

Collect peripheral blood serum to explore the changes of cytokines, Th1 cytokines (IL-1 $\beta$, IL-2, TNF-a, ITN- $\gamma$ ), Th2

cytokines (IL-4, IL-6, IL -10).

2.8 Pregnancy test: blood $\beta-H C G$, female subjects before menopause are examined during the screening period and follow-up period D90 \pm 3 .

2.9 Urine routine: color, clarity, urine sugar, bilirubin, ketone bodies, specific gravity, pH, urobilinogen, nitrite, protein, occult blood, leukocyte enzymes, red blood cells, white blood cells, epithelial cells, non-squamous epithelial cells, Transparent cast, pathological cast, crystal, fungus;

2.10 Stool Routine: color, traits, white blood cells, red blood cells, fat globules, eggs of parasites, fungi, occult blood (chemical method), occult blood (immune method), transferrin ( $2 \mathrm{~h} \pm 30 \mathrm{~min}$ after the injection and not detected after discharge).

Randomization: Block randomization method will be applied by computer to allocate the participants into experimental and control groups. The random ratio is 1:1.

Blinding (masking): Participants, outcomes assessors and investigators (including personnel in laboratory and imaging department who issue the sample report or image observations) will be blinded.

Injections of cell suspension and saline will be coded in accordance with the patient's randomisation group. The blind strategy is kept by an investigator who does not deliver the medical care or assess primary outcome results.

Numbers to be randomized (sample size): Twenty participants will be randomized to the experimental and control groups (10 per group).

Trial Status: Protocol version number, hDPSC-CoVID-2019-02-2020 Version 2.0, March 13, 2020.

Patients screening commenced on $16^{\text {th }}$ April and an estimated date of the recruitment of the final participants will be around end of July. .

Trial registration: Registration:

World Health Organization Trial Registry: ChiCTR2000031319; March 27,2020.

ClinicalTrials.gov Identifier: NCT04336254; April 7, 2020

Other Study ID Numbers: hDPSC-CoVID-2019-02-2020

Full protocol: The full protocol is attached as an additional file, accessible from the Trials website (Additional file 1). In the interest in expediting dissemination of this material, the familiar formatting has been eliminated; this Letter serves as a summary of the key elements of the full protocol.

Keywords: COVID-19; randomised controlled trial; protocol; human dental pulp stem cells; dental stem cell banking 


\section{Supplementary information}

Supplementary information accompanies this paper at https://doi.org/10. 1186/s13063-020-04380-5.

Additional file 1. Full study protocol.

\section{Acknowledgements}

The authors acknowledge the Chu-Tse Wu Foundation for Science \& Technology Development for sponsoring this study. Beijing SH Biotechnology Co., Ltd. and Utooth Biological Technology Co., Ltd. are acknowledged for providing the DPSCs for this study. We would like to extend our appreciation to our collaborators and the nurses, clinicians and lab technicians at the Renmin Hospital and Center of Regenerative Medicine, Wuhan University.

\section{Authors' contributions}

QY, ZW and SLW conceived the research idea; QY, HW, XX, YH and GZ designed the study protocol and developed the research plan; CZ and QY obtained the ethics approval; QY and ZL coordinated the tasks among different investigators; CZ, ZZ, ZL and QY YZ and $\mathrm{KH}$ recruited the participants and collected data. JY and SW will deliver the treatment to the participants; ZX and BW will be the quality controller for the DPSCs intervention; XX manages the blind strategy. QY and YH will analyse the data and interpret the result. YH drafted the manuscript; QY, HW, XX revised and finalised the manuscript; QY, ZW and SLW proofread the manuscript prior to submission. The author(s) read and approved the final manuscript.

\section{Funding}

The trial has been financially funded by the Chu-Tse Wu Foundation for Science \& Technology Development, a non-profit organization. The design of the study, as well as the collection, analysis and interpretation of data will not be influenced by the funding body. The writing of the protocol and the decision to submit for publication are compiled independent from the funding body.

\section{Availability of data and materials}

The data will be stored in a repository managed by a third party called Clinflash, a professional data management service for clinical studies (weblink: https://edc.clinflash.net/shbio). Member investigators of the trial will have access to the final trial dataset, depending on level of need-to-know.

\section{Ethics approval and consent to participate}

The study was approved by the Committee of Ethics for Clinical Research, Renmin Hospital of Wuhan University, on 03/14/2020. The ethical approval number is WDRY2020-K106. Recruitment has been ongoing by the investigators. Once informed consent is signed off by both parties, the investigator and participating patient/ legal representative, the participant will be enrolled in the study.

\section{Consent for publication}

The consent form and materials are available from the corresponding author on request.

\section{Competing interests}

The authors declare that they have no competing interests.

\section{Author details}

${ }^{1}$ Renmin Hospital of Wuhan University, Wuhan 430060, Hubei, China. ${ }^{2}$ Beijing Institute of Radiation Medicine, Beijing 100850, China. ${ }^{3}$ Beijing SH Biotechnology Co., Ltd., Beijing 100070, China. ${ }^{4}$ Tianjin Fopcells

Pharmaceutical Technology Co., Ltd, Tianjin 300074, China. ${ }^{5}$ School of Stomatology, Capital Medical University, Beijing 100006, China. ${ }^{6}$ Tianyou Hospital, Wuhan University of Science and Technology, Wuhan 430064, Hubei, China. ${ }^{7}$ Utooth Biological Technology Co., Ltd., Hubei Branch, Wuhan 430060, Hubei, China.

Received: 24 April 2020 Accepted: 5 May 2020

Published online: 12 June 2020

\section{Publisher's Note}

Springer Nature remains neutral with regard to jurisdictional claims in published maps and institutional affiliations.
Ready to submit your research? Choose BMC and benefit from:
- fast, convenient online submission
- thorough peer review by experienced researchers in your field
- rapid publication on acceptance
- support for research data, including large and complex data types
- gold Open Access which fosters wider collaboration and increased citations
- maximum visibility for your research: over $100 \mathrm{M}$ website views per year
At BMC, research is always in progress.
Learn more biomedcentral.com/submissions

